\title{
EFEITO DA IDADE SOBRE O POLINÔMIO DO QUINTO GRAU PARA AFILAMENTO DE Pinus taeda L.
}

\section{EFFECT OF AGE ON THE FIFTH DEGREE POLYNOMIAL FOR TAPER OF Pinus taeda L.}

\author{
Saulo Jorge Téo ${ }^{1}$, João Henrique Esteves ${ }^{2}$ \\ 1, 2 Universidade do Oeste de Santa Catarina, UNOESC, Xanxerê, Santa Catarina, Brasil - \\ saulo.teo@unoesc.edu.br, jaoesteves@hotmail.com
}

RESUMO

\begin{abstract}
A forma dos troncos das árvores é um importante parâmetro de qualidade e necessária para calcular e estimar o volume dos troncos das árvores, contudo varia conforme uma série de fatores, entre eles a idade das árvores. 0 objetivo deste estudo é demonstrar o efeito da idade sobre os parâmetros do polinômio do quinto grau e, consequentemente, sobre a forma do tronco de árvores de Pinus taeda L., visando a formulação de funções de afilamento, que podem representar troncos de diferentes formas. Os dados são provenientes de 631 árvores, com idades variando de 4 a 31 anos, de diversos povoamentos florestais na região Meio Oeste do estado de Santa Catarina, Brasil. Foi ajustado e testado o polinômio do quinto grau sem qualquer alteração, e com seus coeficientes expressos como função linear da idade, por meio do procedimento PROC NLIN do aplicativo computacional SAS ${ }^{\circledR}$ OnDemand for Academics. O polinômio do quinto grau, com os coeficientes $b_{1}$ e $b_{5}$ ajustados como função linear da idade, apresentou o melhor desempenho estatístico, realismo biológico e conformidade com o conhecimento teórico sobre a forma dos troncos das árvores de Pinus taeda. Dessa forma, apenas uma equação de afilamento foi capaz de estimar diâmetros ao longo do tronco de árvores de diferentes formas, com precisão e exatidão.
\end{abstract}

PALAVRAS-CHAVE: Forma, Função de afilamento, Regressão não linear.

\section{ABSTRACT}

The stem taper of the trees is an important quality parameter and necessary to calculate and estimate the volume of the tree stems, however it varies according to a series of attributes, including the age of the trees. The objective of this research is to demonstrate the effect of age on the parameters of the fifth-degree polynomial and, consequently, on the stem taper of Pinus taeda L. trees, aiming at the formulation of taper functions, which can represent stems of different tapering. The data came from 631 trees, with ages ranging from 4 to 31 years, from different forest stands in the Midwest region of the state of Santa Catarina, Brazil. The fifth-degree polynomial was adjusted and tested without any change, and with its coefficients expressed as a linear function of age, using the PROC NLIN procedure of the computer application SAS ${ }^{\circledR}$ OnDemand for Academics. The fifth-degree polynomial, with the coefficients $b_{1}$ and $b_{5}$ expressed as a linear function of age, presented the best statistical performance, biological consistency, and conformity with theoretical knowledge about the stem taper of Pinus taeda trees. Thus, only one taper equation was able to estimate diameters along the stem of trees of different tapering, with precision and accuracy.

KEYWORDS: Form, Taper function, Nonlinear regression. 


\section{INTRODUÇÃO}

A forma do fuste das árvores diz respeito à figura ou contorno externo do tronco, geralmente apresentando afilamento da base até o topo. Segundo Husch et al. (2003), a forma do fuste é definida pelo processo de crescimento do diâmetro e da área seccional ao longo do tronco da árvore. Não se pode medir a forma de uma árvore ou tora como se faz com o diâmetro ou a altura, somente estabelecer índices, ou ajustar funções que a representam. A captação da forma dos troncos das árvores torna-se possível pela medição de diâmetros a distintas alturas, e sua expressão se dá, principalmente, por meio de quocientes de forma, fatores de forma e funções de afilamento.

A forma é um dos mais importantes parâmetros externos de qualidade do fuste, frequentemente utilizado como critério de sortimento florestal. Fustes com forma inferior, tais como aqueles que apresentam afilamento e curvatura acentuados, são usualmente relacionados com baixo rendimento em serrarias, propriedades mecânicas da madeira ruins e alto custo de processamento (TONG; ZHANG, 2008). As equações de afilamento, além de expressar a forma dos troncos das árvores, podem fornecer estimativas de diâmetros a diferentes alturas, altura onde se encontra um dado diâmetro, volume total e volumes comerciais de madeira.

Todas as árvores apresentam uma forma típica de seu fuste, a qual varia com a espécie, idade, sítio, posição sociológica, densidade do povoamento, intervenções silviculturais como desbaste e poda, e até estresses mecânicos, como os causados pelo efeito do vento sobre a árvore (AHRENS \& HOLBERT, 1981). Dessa forma, usualmente, o desenvolvimento de modelos de afilamento se restringe a uma única espécie, classe de idade, regime de manejo, qualidade de sítio florestal, região de estudo e, até mesmo, classes de diâmetro, classes de altura e classes de fator de forma ( FIGUEIREDO et al., 2006; EISFELD et al., 2008; SOUZA et al., 2008; MIGUEL et al., 2011; FAVALESSA et al., 2012a; FAVALESSA et al., 2012b; SOUZA et al., 2012; KOHLER et al., 2013; LANSSANOVA et al., 2013; TÉO et al., 2013; DAVID et al., 2014; FIGUEIREDO FILHO et al., 2014; FIGUEIREDO FILHO et al., 2015; COSTA et al., 2016; KOHLER et al., 2016; TÉO et al., 2018b; TERRA et al., 2018).

Alguns autores recomendaram o ajuste de funções de afilamento por classe de idade e forma (KOHLER et al., 2013; DAVID et al., 2014; FIGUEIREDO FILHO et al., 2015; KOHLER et al., 2016; TERRA et al., 2018), porém este procedimento gera tantas equações quantas forem as classes em que os dados forem estratificados. Caso o número de equações seja muito grande, haverá menor eficiência e produtividade no momento de sua aplicação, visando produzir estimativas.

Para evitar esse problema e desenvolver uma equação de afilamento de maior aplicabilidade, Téo (2017) e Téo et al. (2018a) desenvolveram funções de afilamento, cujos parâmetros foram expressos como combinação linear da idade das árvores, já que a idade é uma das variáveis que mais interfere na forma dos troncos das árvores. Essas funções de afilamento, desenvolvidas por Téo (2017) e Téo et al. (2018a) não mostraram melhoras expressivas de precisão e exatidão das estimativas de diâmetros, quando comparadas às funções de afilamento em suas formulações simples.

Os autores deste estudo acreditam que os resultados encontrados por Téo (2017) e Téo et al. (2018a) podem ser atribuídos ao fato de somente um dos parâmetros das funções de afilamento terem sido expressos como função linear da idade de cada vez. Dessa forma, a hipótese deste estudo é que melhores resultados podem ser obtidos caso essa mesma técnica de ajuste seja realizada com mais de um parâmetro da função de afilamento. Dessa forma, o objetivo deste estudo é demonstrar o efeito da idade sobre os parâmetros do polinômio do quinto grau e, consequentemente, sobre o perfil do tronco de árvores de Pinus taeda L., visando contribuir com as técnicas de ajuste e formulação de funções de afilamento, que poderão representar troncos de diferentes formas.

\section{MATERIAL E MÉTODOS}

Os dados para realização desta pesquisa foram coletados, em povoamentos de Pinus taeda pertencentes à empresa Juliana Florestal Ltda., associada à FRAME Madeiras Especiais Ltda., sediada em Caçador, Santa Catarina. Além de Caçador, a área de estudo compreende os municípios de Calmon, Lebon Régis, Macieira, Rio das Antas, Santa Cecília e Timbó Grande, todos na região Meio Oeste do estado de Santa Catarina.

Segundo a classificação de Köppen, a região de estudo possui clima tipo $\mathrm{Cfb}$, isto é, da zona subtropical húmida, clima oceânico, sem estação seca e com verões temperados. A temperatura média do mês mais quente é $19,7^{\circ} \mathrm{C}$ e do mês mais frio $11,5^{\circ} \mathrm{C}$, a precipitação anual é de $1736 \mathrm{~mm}$ (ALVARES et al., 2013). A vegetação original da região de estudo é a Floresta Ombrófila Mista, principalmente em sua formação Floresta Ombrófila Mista Montana. Os principais solos da área de estudo são Cambissolos háplicos, com grande variação de profundidade e drenagem variando de acentuada à imperfeita e, Nitossolos brunos de textura argilosa ou muito argilosa, moderadamente ácidos (IBGE, 2007). 
Os dados são provenientes de 631 árvores de Pinus taeda, com idades variando de 4 a 31 anos, de diversos povoamentos distribuídos na área de estudo. Desse total, 519 árvores foram abatidas para a realização do procedimento de cubagem de Smalian, as 112 árvores restantes tiveram os seus diâmetros com casca medidos ao longo do fuste, utilizando de maneira combinada o Criterion RD 1000 e o TruPulse 200B, os quais permitiram a obtenção de medições para o procedimento de cubagem de Smalian de maneira não destrutiva, ou seja, sem o abate das árvores. Tais equipamentos foram utilizados visando a diminuição do número de árvores derrubadas, assim, reduzindo os custos da coleta de dados para volumetria das árvores de Pinus taeda.

Para as 519 árvores de Pinus taeda abatidas, foram tomadas medições de diâmetro com casca a $0 \%, 0,5 \%, 1 \%$, $5 \%, 10 \%, 15 \%, 20 \%, 25 \%, 30 \%, 40 \%, 50 \%, 60 \%, 70 \%, 80 \%$, $90 \%$ e $95 \%$ da altura total da árvore. Para as 112 árvores medidas em pé, com o Criterion RD 1000 e o TruPulse 200B, os diâmetros com casca foram tomados nas mesmas posições relativas à altura total da árvore, até, no mínimo, $70 \%$ e foi considerada a altura de toco média de 0,1 $\mathrm{m}$.

A Tabela 1 apresenta a distribuição das 631 árvores de Pinus taeda cubadas, nas diferentes idades $(t)$, bem como, os valores de diâmetro médio $(\bar{d})$, diâmetro máximo $\left(d_{\text {máx }}\right)$, diâmetro mínimo $\left(d_{m i ́ n}\right)$, altura média $(\bar{h})$, altura máxima $\left(h_{m a ́ x}\right)$ e mínima $\left(h_{m i ́ n}\right)$.

Para todo o conjunto de dados, foi ajustado o polinômio do quinto grau sem qualquer alteração, e com seus coeficientes expressos como função linear da idade, por meio do procedimento PROC NLIN do software SAS ${ }^{\circledR}$ OnDemand for Academics (Tabela 2).

Tabela 1. Diâmetro à altura do peito $(1,3 \mathrm{~m})(\mathrm{cm})$, altura total $(\mathrm{m})$ e número de árvores de Pinus taeda L., por classe de idade (anos).

\begin{tabular}{cccccccc}
\hline $\boldsymbol{t}$ & $\mathbf{n}$ & $\overline{\boldsymbol{d}}$ & $\boldsymbol{d}_{\boldsymbol{m a ́ x}}$ & $\boldsymbol{d}_{\boldsymbol{m i n}}$ & $\overline{\boldsymbol{h}}$ & $\boldsymbol{h}_{\text {máx }}$ & $\boldsymbol{h}_{\boldsymbol{m i n}}$ \\
\hline 4 & 30 & 8,60 & 14,50 & 3,90 & 5,28 & 7,40 & 3,10 \\
\hline 5 & 63 & 12,80 & 21,00 & 3,70 & 7,65 & 10,20 & 3,37 \\
\hline 6 & 89 & 13,43 & 23,00 & 2,90 & 8,37 & 12,70 & 4,08 \\
\hline 7 & 60 & 15,19 & 23,10 & 3,90 & 9,77 & 12,80 & 5,10 \\
\hline 8 & 30 & 16,25 & 26,00 & 4,00 & 12,41 & 16,25 & 5,17 \\
\hline 9 & 30 & 19,66 & 32,00 & 7,70 & 13,98 & 16,80 & 7,90 \\
\hline 10 & 59 & 19,69 & 32,50 & 6,00 & 14,71 & 17,80 & 8,30 \\
\hline 11 & 30 & 19,60 & 31,70 & 8,20 & 14,81 & 18,80 & 9,22 \\
\hline 12 & 39 & 23,53 & 35,50 & 12,50 & 18,57 & 22,60 & 14,90 \\
\hline 14 & 29 & 23,95 & 36,92 & 13,37 & 18,88 & 21,70 & 15,80 \\
\hline 16 & 23 & 22,99 & 37,56 & 15,60 & 19,45 & 22,30 & 16,20 \\
\hline 18 & 37 & 28,07 & 48,06 & 15,92 & 23,38 & 30,20 & 17,30 \\
\hline 21 & 65 & 35,00 & 49,97 & 21,65 & 28,98 & 33,00 & 21,50 \\
\hline 26 & 13 & 26,98 & 43,90 & 17,50 & 27,69 & 29,50 & 25,00 \\
\hline 27 & 12 & 29,31 & 38,83 & 21,96 & 28,87 & 32,80 & 25,40 \\
\hline 30 & 12 & 35,83 & 51,80 & 22,60 & 29,23 & 32,00 & 27,30 \\
\hline 31 & 10 & 32,60 & 44,20 & 25,10 & 31,08 & 35,00 & 25,00 \\
\hline
\end{tabular}

Tabela 2. Modelos de afilamento testados para estimar o diâmetro com casca, de árvores de Pinus taeda L.

\begin{tabular}{ll}
\hline $\mathbf{n}$ & $d_{i}=d\left\{b_{0}+b_{1}\left(\frac{h_{i}}{h}\right)+b_{2}\left(\frac{h_{i}}{h}\right)^{2}+b_{3}\left(\frac{h_{i}}{h}\right)^{3}+b_{4}\left(\frac{h_{i}}{h}\right)^{4}+b_{5}\left(\frac{h_{i}}{h}\right)^{5}\right\}$ \\
\hline 1 & $d_{i}=d\left\{\left[b_{01}+b_{02} t\right]+b_{1}\left(\frac{h_{i}}{h}\right)^{2}+b_{2}\left(\frac{h_{i}}{h}\right)^{2}+b_{3}\left(\frac{h_{i}}{h}\right)^{3}+b_{4}\left(\frac{h_{i}}{h}\right)^{4}+b_{5}\left(\frac{h_{i}}{h}\right)^{5}\right\}$ \\
3 & $d_{i}=d\left\{b_{0}+\left[b_{11}+b_{12} t\right]\left(\frac{h_{i}}{h}\right)^{2}+b_{2}\left(\frac{h_{i}}{h}\right)^{2}+b_{3}\left(\frac{h_{i}}{h}\right)^{3}+b_{4}\left(\frac{h_{i}}{h}\right)^{4}+b_{5}\left(\frac{h_{i}}{h}\right)^{5}\right\}$ \\
4 & $d_{i}=d\left\{b_{0}+b_{1}\left(\frac{h_{i}}{h}\right)+\left[b_{21}+b_{22} t\right]\left(\frac{h_{i}}{h}\right)^{2}+b_{3}\left(\frac{h_{i}}{h}\right)^{3}+b_{4}\left(\frac{h_{i}}{h}\right)^{4}+b_{5}\left(\frac{h_{i}}{h}\right)^{5}\right\}$ \\
6 & $d_{i}=d\left\{b_{0}+b_{1}\left(\frac{h_{i}}{h}\right)+b_{2}\left(\frac{h_{i}}{h}\right)^{2}+\left[b_{31}+b_{32} t\right]\left(\frac{h_{i}}{h}\right)^{3}+b_{4}\left(\frac{h_{i}}{h}\right)^{4}+b_{5}\left(\frac{h_{i}}{h}\right)^{5}\right\}$ \\
7 & $d_{i}=d\left\{b_{0}+b_{1}\left(\frac{h_{i}}{h}\right)+b_{2}\left(\frac{h_{i}}{h}\right)^{2}+b_{3}\left(\frac{h_{i}}{h}\right)^{3}+\left[b_{41}+b_{42} t\right]\left(\frac{h_{i}}{h}\right)^{4}+b_{5}\left(\frac{h_{i}}{h}\right)^{5}\right\}$ \\
8 & $d_{i}=d\left\{b_{0}+b_{1}\left(\frac{h_{i}}{h}\right)+b_{2}\left(\frac{h_{i}}{h}\right)^{2}+b_{3}\left(\frac{h_{i}}{h}\right)^{3}+b_{4}\left(\frac{h_{i}}{h}\right)^{4}+\left[b_{51}+b_{52} t\right]\left(\frac{h_{i}}{h}\right)^{5}\right\}$
\end{tabular}

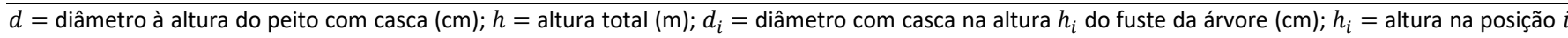
do fuste da árvore $(\mathrm{m}) ; b_{0}, b_{1}, b_{2}, b_{3}, b_{4}, b_{5}=$ coeficientes do polinômio do quinto grau a serem estimados; $b_{01}, b_{02}, b_{11}, b_{12}, b_{21}, b_{22}, b_{31}, b_{32}, b_{41}, b_{42}, b_{51}, b_{52}=$ coeficientes para a função linear da idade; $t=$ idade (anos). 
Para atestar o desempenho dos modelos ajustados foram calculados: coeficiente de correlação ao quadrado $\left(R_{Y \hat{Y}}^{2}\right)$, erro padrão relativo (syx\%), média das diferenças $(M D)(\mathrm{cm})$ e análise dos resíduos studentizados. Os resíduos studentizados foram representados sobre a variável dependente estimada $\left(\hat{Y}_{i}\right)$, almejando-se distribuição de resíduos uniforme, com ausência de padrões e heterocedasticidade. Para verificar se os resíduos apresentam distribuição normal, foi feita a representação gráfica desses valores ordenados crescentemente, sobre os quantis teóricos da distribuição normal. Uma vez detectada heterocedasticidade e não normalidade dos resíduos, foi aplicado o ajuste pelo método dos mínimos quadrados ponderados, conforme Parresol (1993) e Huber (1964), respectivamente. Os modelos foram também submetidos à validação recorrendo-se aos resíduos PRESS $\left(e_{i,-i}\right)$, conforme Myers (1986), calculando-se as estatísticas de validação: eficiência da modelagem $(E M)$; média das diferenças PRESS $\left(M D_{P R E S S}\right)(\mathrm{cm})$; média das diferenças absolutas PRESS $\left(M A D_{P R E S S}\right)(\mathrm{cm})$. Por fim, o modelo de melhor desempenho estatístico e validação, com coeficientes dependentes da idade, foi comparado ao polinômio do 5 으 grau (Modelo 1), por meio dos valores da média das diferenças $(M D)(\mathrm{cm})$ e média das diferenças absolutas $(M D A)(\mathrm{cm})$, calculados por classe de idade e para as diferentes posições relativas da altura da árvore: $0 \%, 0,5 \%$, $1 \%, 5 \%, 10 \%, 15 \%, 20 \%$, 25\%, 30\%, 40\%, 50\%, 60\%, 70\%, $80 \%, 90 \%, 95 \%$ e $100 \%$.

\section{RESULTADOS E DISCUSSÃO}

Todos os modelos de afilamento ajustados para Pinus taeda apresentaram coeficientes significativos $(\alpha=0,05)$ na forma como se encontram na Tabela 2.

O modelo 8 apresentou os melhores critérios estatísticos de seleção $\left(R_{Y \hat{Y}}^{2}, s y x \%, M D\right)$ para estimar os diâmetros com casca ao longo do tronco de Pinus taeda, seguido dos modelos 3 e 4 (Tabela 3). Considerando todos os critérios estatísticos de validação, o modelo 8 também apresentou o melhor desempenho, seguido pelo modelo 1 , que apresentou menor viés $\left(M D_{P R E S S}\right)$, e pelo modelo 3 (Tabela 3). É possível notar que os valores dos critérios de seleção e validação foram muito semelhantes para todos os modelos de afilamento testados, com pequena superioridade para o modelo 8 (Tabela 3).

Optou-se por apresentar apenas a distribuição de resíduos studentizados para os modelos 3 e 8 . No entanto, a distribuição de resíduos do Modelo 3 foi muito semelhante aos modelos 4, 5, 6 e 7 e a distribuição de resíduos do Modelo 8 foi muito semelhante aos Modelos 1 e 2 (Figura 1).

Tabela 3. Critérios de seleção e validação dos modelos de afilamento do tronco com casca de Pinus taeda L.

\begin{tabular}{cccc}
\hline \multicolumn{4}{c}{ Critérios de Seleção } \\
\hline Modelo & $\boldsymbol{R}_{\boldsymbol{Y} \widehat{\boldsymbol{Y}}}^{2}$ & $\boldsymbol{s y \boldsymbol { x }} \%$ & $\boldsymbol{M D}$ \\
\hline 1 & 0,98193 & 9,82 & 0,14307 \\
2 & 0,98204 & 9,81 & 0,15216 \\
3 & 0,98385 & 9,46 & 0,20722 \\
4 & 0,98335 & 9,55 & 0,18940 \\
5 & 0,98285 & 9,65 & 0,17607 \\
6 & 0,98253 & 9,71 & 0,16750 \\
7 & 0,98234 & 9,75 & 0,16182 \\
8 & 0,98474 & 9,31 & 0,12514 \\
\hline \multicolumn{4}{c}{ Critérios de Validação } \\
\hline Modelo & $\boldsymbol{E M}$ & $\boldsymbol{M} \boldsymbol{D}_{\boldsymbol{P R E S S}}$ & $\boldsymbol{M} \boldsymbol{A} \boldsymbol{D}_{\boldsymbol{P R E S S}}$ \\
\hline 1 & 0,98140 & 0,14252 & 1,08780 \\
2 & 0,98143 & 0,15484 & 1,08934 \\
3 & 0,98272 & 0,21294 & 1,08056 \\
4 & 0,98241 & 0,19381 & 1,08827 \\
5 & 0,98204 & 0,17909 & 1,09501 \\
\hline 6 & 0,98180 & 0,16946 & 1,09749 \\
7 & 0,98166 & 0,16300 & 1,09800 \\
8 & 0,98332 & 0,18074 & 1,03365 \\
\hline
\end{tabular}
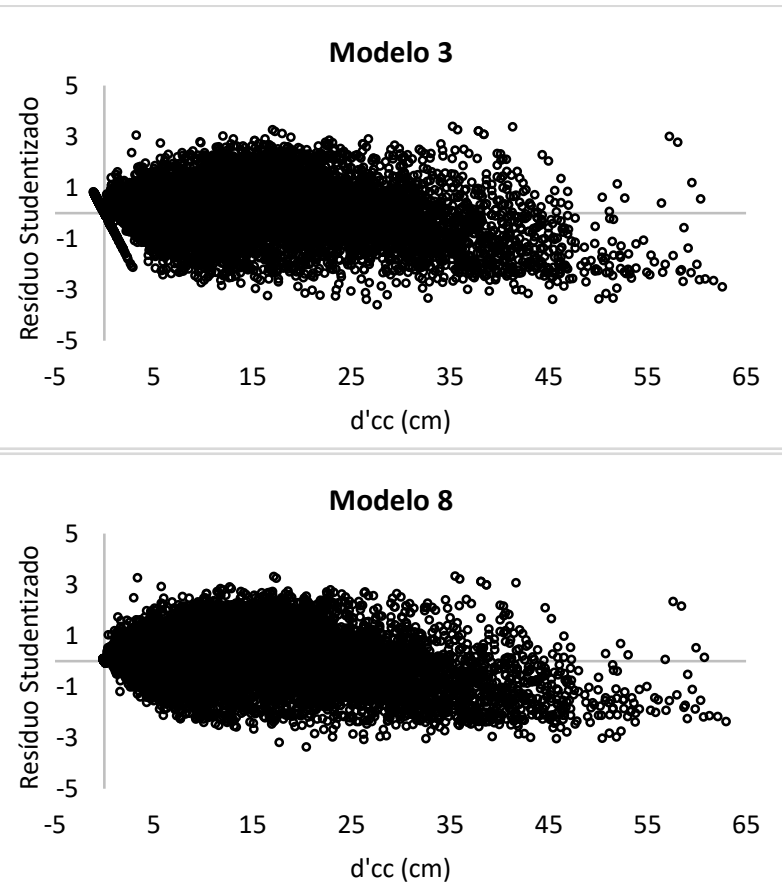

Figura 1. Distribuição de resíduos studentizados para os modelos 3 e 8.

A distribuição de resíduos studentizados foi semelhante para todos os modelos de afilamento testados, sem heterocedasticidade e seguindo a distribuição normal. Para todos os modelos de afilamento ajustados, há maior 
frequência de valores negativos de resíduos studentizados para os maiores valores de diâmetro com casca estimados $\left(d_{c c}^{\prime}\right)$, podendo indicar tendência de superestimativa. No entanto, o modelo 3 apresentou um padrão nos resíduos studentizados calculados para a posição da altura total da árvore $\left(h_{i} / h=1\right)$, onde os diâmetros observados são iguais a zero (Figura 1). Esse mesmo padrão na distribuição dos resíduos studentizados também ocorreu para os modelos 4, 5, 6 e 7.

Para demonstrar o efeito da idade sobre os coeficientes do polinômio do quinto grau foram confeccionados os perfis do tronco com casca das árvores de Pinus taeda, por meio de todos os modelos de afilamento ajustados, para as diferentes idades dentro da amplitude de variação dos dados utilizados para o ajuste (Figuras 2 e 3 ).
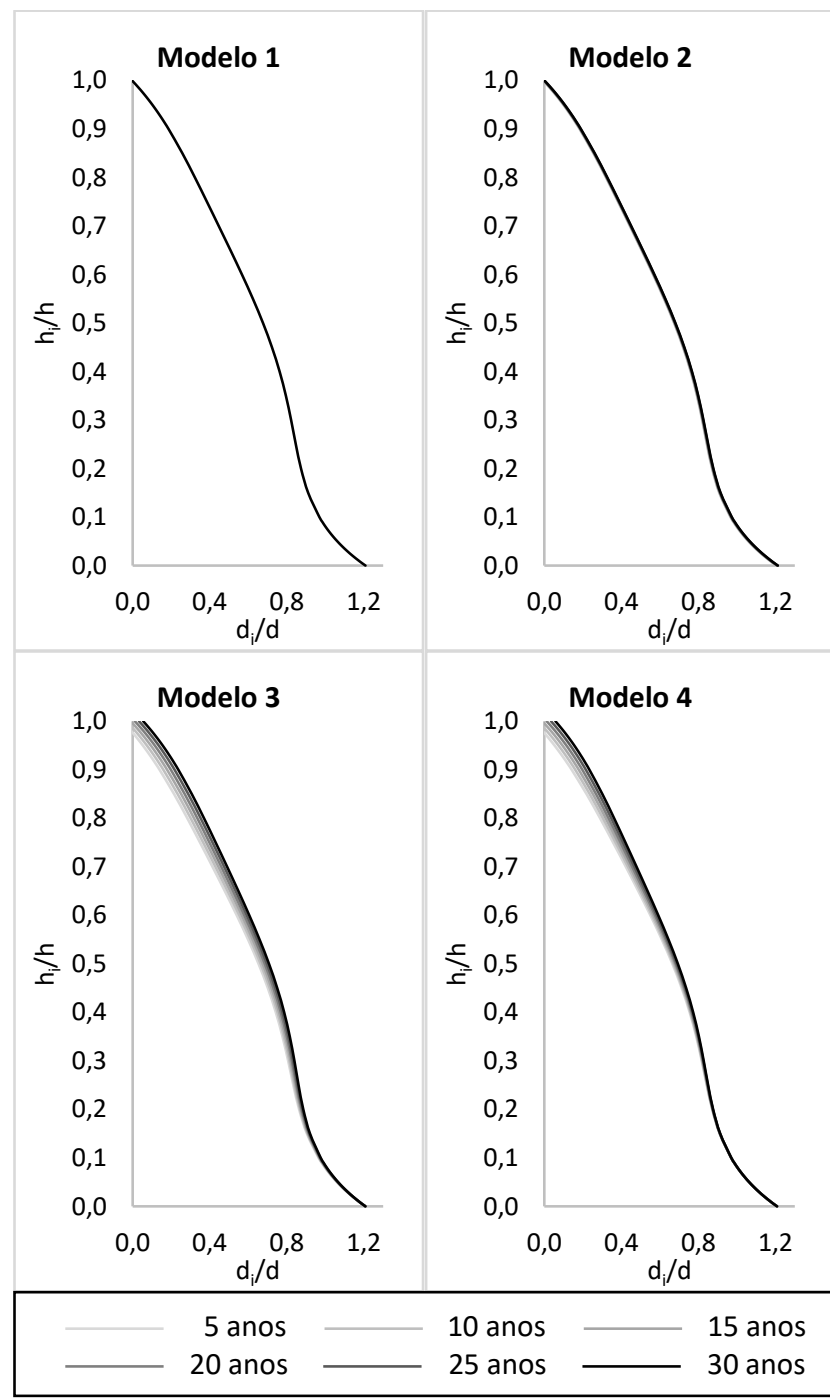

Figura 2. Perfil do tronco com casca para árvores de Pinus taeda L., resultante dos modelos 1, 2, 3 e 4.

O modelo 1 apresenta apenas um perfil do tronco médio para árvores de Pinus taeda de todas as idades, uma vez que a idade não é covariável desse modelo. O modelo
2 , apesar de ter o coeficiente $b_{0}$ ajustado como função linear da idade, não apresentou diferenças perceptíveis no perfil do tronco conforme as idades (5 a 30 anos) (Figura 2). Assim, o perfil do tronco resultante do modelo 2 foi muito semelhante ao perfil do troco resultante do polinômio do quinto grau sem qualquer alteração (Modelo 1).

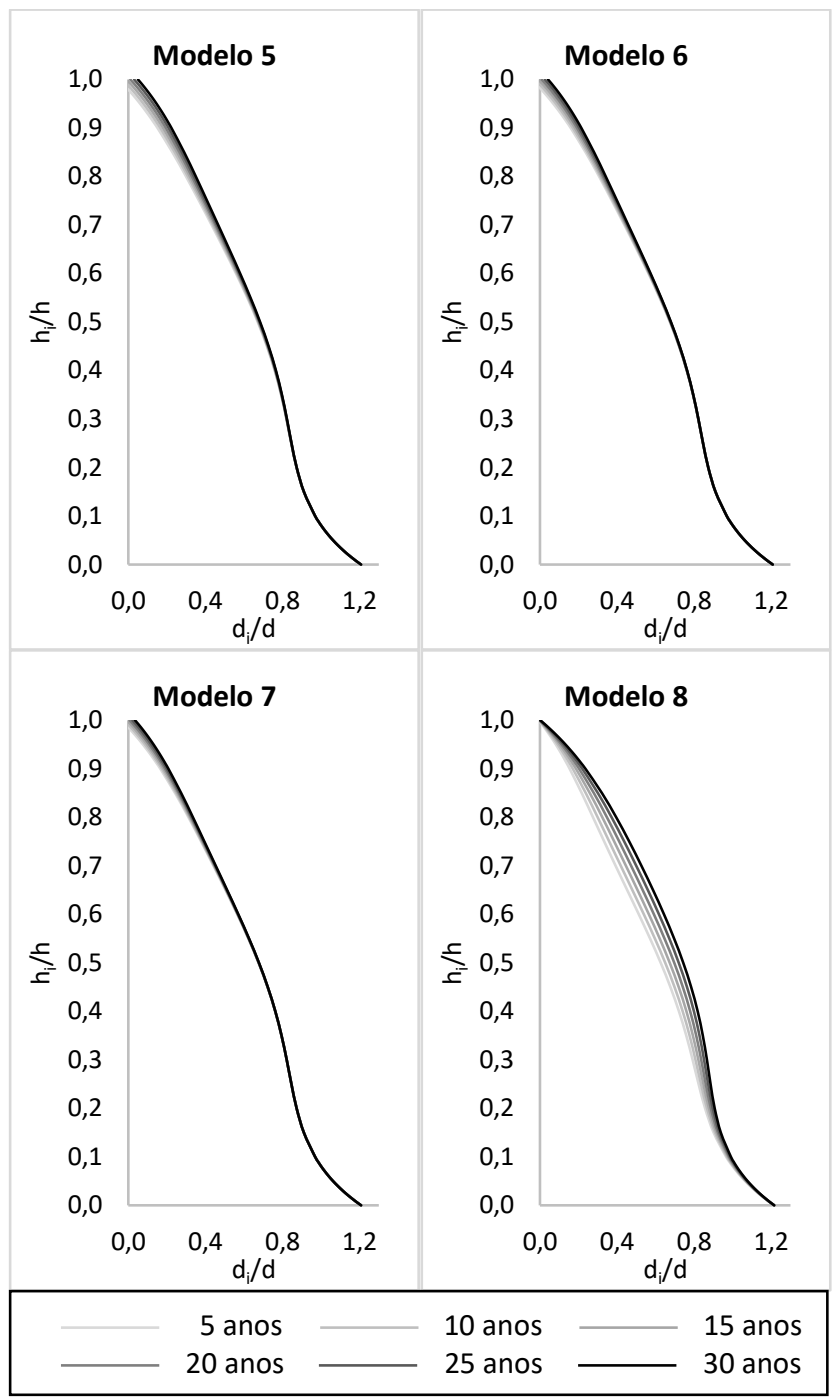

Figura 3. Perfil do tronco com casca para árvores de Pinus taeda L., resultante dos modelos 5, 6, 7 e 8.

O modelo 3, com o coeficiente $b_{1}$ ajustado como função linear da idade, apresentou diferenças no perfil do tronco das árvores conforme as idades, desde a porção basal do perfil do tronco (Figura 2). Os perfis do tronco gerados a partir do modelo 3 ao 7 apresentam diâmetros com casca ao longo do tronco das árvores tanto maiores, quanto maior a idade das árvores. Para o modelo 3, é possível visualizar essa tendência a partir de $20 \%$ da altura total das árvores de Pinus taeda (Figura 2). No entanto, a partir dessa posição, os perfis do tronco não mais diferem conforme as idades, gerando estimativas de diâmetros na 
posição da altura total da árvore $\left(h_{i} / h=1\right)$ negativos para as menores idades e positivos para as maiores idades. Esse comportamento do modelo 3 explica o padrão na dispersão dos resíduos studentizados calculados para a posição $h_{i} / h=1$, onde os diâmetros observados são iguais a zero (Figura 1 ).

Os Modelos 4, 5, 6 e 7, com os coeficientes $b_{2}, b_{3}, b_{4}$ e $b_{5}$ ajustados como função linear da idade, respectivamente, apresentaram diferenças no perfil do tronco das árvores conforme as idades, semelhante ao ocorrido para o modelo 3 . É possível perceber que, do modelo 3 ao modelo 7, o efeito da idade sobre os perfis do tronco passa da porção inferior para a porção superior. Todavia, sempre com tendência de apresentar estimativas do diâmetro ao longo do tronco maiores, para as maiores idades (Figuras 2 e 3). Essa tendência ocorre para o modelo 7, somente na porção superior do perfil do tronco, a partir de $80 \%$ da altura total das árvores de Pinus taeda (Figura 3).

O modelo 8, com os coeficientes $b_{1}$ e $b_{5}$ ajustados como função linear da idade, apresentou efeito nos perfis do tronco das árvores de Pinus taeda, tanto na porção inferior como na porção superior do tronco. A partir de, aproximadamente, $10 \%$ da altura total das árvores, há tendência de diâmetros ao longo do tronco tanto maiores, quanto maior a idade das árvores. Por outro lado, o oposto pode ser observado a partir de, aproximadamente, $80 \%$ da altura total das árvores (Figura 3). Com isso, as estimativas de diâmetros na posição da altura total da árvore $\left(h_{i} / h=\right.$ 1) não apresentaram o problema descrito para os Modelos 3 ao 7, que resultaram em estimativas de diâmetros na posição da altura total da árvore $\left(h_{i} / h=1\right)$ negativos para as menores idades e positivos para as maiores idades e, além disso, causaram padrões na dispersão dos resíduos studentizados (Figuras 1, 2 e 3).

Devido aos coeficientes $b_{1}$ e $b_{5}$ ajustados como função linear da idade, o modelo 8 foi capaz de gerar perfis do tronco com a porção intermediária mais cônicas para as árvores mais jovens e, com formas mais próximas ao cilindro, para as árvores mais velhas (Figura 3). A série de perfis do tronco gerada pelo modelo 8 , para árvores de Pinus taeda de diferentes idades (Figura 3), está de acordo com a evolução da forma das árvores com relação à idade. Diversos estudos indicam que a forma do tronco das árvores coníferas altera-se com a idade, sendo que o afilamento diminui com o avanço da idade, ou seja, os troncos tornam-se menos cônicos e mais cilíndricos (KOHLER et al., 2013; TÉO et al., 2013; FIGUEIREDO FILHO et al., 2014; FIGUEIREDO FILHO et al., 2015; KOHLER et al., 2016; MARTINS et al., 2017).

Para a maioria das classes de idade de Pinus taeda, o modelo 8 apresentou menor viés $(M D)$ e maior precisão $(M D A)$ do que o modelo 1 (Tabela 4). Esses resultados indicam melhor desempenho estatístico do modelo 8 para estimar os diâmetros com casca ao longo do tronco das árvores de Pinus taeda, por classe de idade.

Tabela 4. Média das diferenças $(M D)$ e média das diferenças absolutas $(M D A)$, por classe de idade e altura relativa, para os modelos de afilamento 1 e 8 .

\begin{tabular}{|c|c|c|c|c|}
\hline \multirow{2}{*}{ Idade } & \multicolumn{2}{|c|}{ Modelo 1} & \multicolumn{2}{|c|}{ Modelo 8} \\
\hline & $M D$ & $M D A$ & $M D$ & $M D A$ \\
\hline 4 & 0,6619 & 1,1290 & 0,8606 & 1,0948 \\
\hline 5 & 0,5925 & 0,9774 & 0,8638 & 0,9759 \\
\hline 6 & 0,3358 & 0,9781 & 0,5885 & 0,8946 \\
\hline 7 & 0,1831 & 0,9651 & 0,4309 & 0,8453 \\
\hline 8 & $-0,4434$ & 0,9549 & $-0,2275$ & 0,7923 \\
\hline 9 & $-0,3968$ & 1,0684 & $-0,1768$ & 0,8889 \\
\hline 10 & 0,0296 & 0,8244 & 0,2088 & 0,8420 \\
\hline 11 & $-0,2617$ & 0,9783 & $-0,1291$ & 0,9243 \\
\hline 12 & $-0,7558$ & 1,2319 & $-0,6520$ & 1,1847 \\
\hline 14 & 0,3391 & 0,9470 & 0,3373 & 0,9900 \\
\hline 16 & 0,2298 & 0,9048 & 0,1193 & 0,9139 \\
\hline 18 & 0,2930 & 1,1822 & 0,0255 & 1,1497 \\
\hline 21 & $-0,1780$ & 1,3552 & $-0,7598$ & 1,4161 \\
\hline 26 & 1,1760 & 1,6856 & 0,4087 & 1,3838 \\
\hline 27 & 0,6007 & 1,4586 & $-0,3021$ & 1,2008 \\
\hline 30 & 0,8539 & 1,8849 & $-0,5035$ & 1,5210 \\
\hline 31 & 0,1977 & 1,5635 & $-1,1145$ & 1,5361 \\
\hline \multirow{2}{*}{$\boldsymbol{h}_{\boldsymbol{i}} / \boldsymbol{h}$} & \multicolumn{2}{|c|}{ Modelo 1} & \multicolumn{2}{|c|}{ Modelo 8} \\
\hline & $M D$ & $M D A$ & $M D$ & $M D A$ \\
\hline 0,000 & 0,4655 & 1,3859 & 0,3116 & 1,3793 \\
\hline 0,005 & 0,8370 & 1,4492 & 0,6817 & 1,4130 \\
\hline 0,010 & 0,3377 & 1,3076 & 0,1812 & 1,3259 \\
\hline 0,050 & $-0,2218$ & 1,3307 & $-0,3754$ & 1,4304 \\
\hline 0,100 & 0,3100 & 1,0395 & 0,1829 & 1,1476 \\
\hline 0,150 & 0,5256 & 0,9912 & 0,4402 & 1,0673 \\
\hline 0,200 & 0,5175 & 1,0010 & 0,4800 & 1,0786 \\
\hline 0,250 & 0,2815 & 0,9549 & 0,2918 & 1,0363 \\
\hline 0,300 & 0,0190 & 0,9244 & 0,0721 & 1,0046 \\
\hline 0,400 & $-0,3052$ & 1,0485 & $-0,1918$ & 0,9856 \\
\hline 0,500 & $-0,2904$ & 1,2550 & $-0,1592$ & 1,0259 \\
\hline 0,600 & $-0,0434$ & 1,4173 & 0,0695 & 1,0531 \\
\hline 0,700 & 0,0813 & 1,5536 & 0,1593 & 1,1189 \\
\hline 0,800 & 0,0071 & 1,2446 & 0,0263 & 0,9309 \\
\hline 0,900 & $-0,1606$ & 0,8109 & $-0,1821$ & 0,7248 \\
\hline 0,950 & 0,0922 & 0,5371 & 0,0782 & 0,5668 \\
\hline 1,000 & 0,0637 & 0,0637 & 0,0695 & 0,0758 \\
\hline
\end{tabular}

Embora o modelo 8 tenha apresentado melhor desempenho estatístico para a maioria das classes de idade, convém ressaltar que apresentou maior viés $(M D)$ para estimativa de diâmetros ao longo do tronco de árvores abaixo de 8 anos. 
Quanto ao viés $(M D)$ precisão $(M D A)$ das estimativas de diâmetro calculados para as diferentes alturas relativas do tronco das árvores de Pinus taeda, os Modelos 1 e 8 foram equivalentes e com valores relativamente semelhantes (Tabela 4). No entanto, é possível perceber a maior precisão $(M D A)$ das estimativas de diâmetro com casca realizadas por meio do modelo 8 , acima de $30 \%$ da altura total das árvores de Pinus taeda.

Considerando o melhor desempenho quanto aos critérios estatísticos de seleção e validação (Tabela 3), a distribuição de resíduos studentizados (Figura 1), o realismo biológico e conformidade com o conhecimento biométrico sobre a forma dos troncos das árvores coníferas (Figura 3), bem como o melhor desempenho estatístico por classe de idade (Tabela 4), a equação de afilamento indicada para troncos de Pinus taeda é resultante do Modelo 8 (Expressão 1).

$$
d_{i}=d\left\{1,2157+[-3,7117+0,0100 t]\left(\frac{h_{i}}{h}\right)+14,4899\left(\frac{h_{i}}{h}\right)^{2}-31,2113\left(\frac{h_{i}}{h}\right)^{3}+29,5059\left(\frac{h_{i}}{h}\right)^{4}+[-10,2966-0,0096 t]\left(\frac{h_{i}}{h}\right)^{5}\right\}
$$

Em que: $d, h, d_{i}, h_{i}, t=$ já definidos anteriormente.

Embora tenha sido a equação indicada neste estudo, os autores não recomendam a utilização da equação de afilamento para troncos de Pinus taeda, fora dos limites de variação de idades do conjunto de dados utilizados para o ajuste (Tabela 1). Essa prática pode gerar perfis do tronco sem realismo biológico, caso idades muito avançadas sejam inseridas na Expressão 1. Para corrigir esse problema, transformações da variável idade $(t)$ poderão ser testadas em trabalhos futuros.

A técnica de ajuste do polinômio do quinto grau, com os coeficientes $b_{1}$ e $b_{5}$ como função linear da idade, permitiu a recomendação de apenas uma equação de afilamento para troncos de Pinus taeda de 4 a 31 anos de idade. Diferentemente de outros estudos que recomendaram o ajuste de funções de afilamento por classe de idade, por apresentarem melhor desempenho estatístico para estimativa dos diâmetros ao longo do tronco (KOHLER et al., 2013; FIGUEIREDO FILHO et al., 2015; KOHLER et al., 2016; MARTINS et al., 2017). No entanto, Kohler et al. (2013), Figueiredo Filho et al. (2015), Kohler et al. (2016) e Martins et al. (2017) testaram o polinômio do quinto grau (modelo 1 ) e o polinômio de potências inteiras e fracionárias de Hradetzky sem o efeito da idade nos seus coeficientes.

As funções de afilamento polinomiais do quinto grau e de potências inteiras e fracionárias de Hradetzky são muito utilizadas pelas empresas de base florestal e muito testadas em estudos recentes sobre o tema (STEPKA et al., 2017; SILVA et al., 2018; TERRA et al., 2018; NICOLETTI et al., 2020). Os autores deste estudo acreditam que a técnica de ajuste com os coeficientes do modelo de afilamento expressos como função linear da idade também poderá ser testada em trabalhos futuros, para polinômio de potências inteiras e fracionárias de Hradetzky. Dessa forma, a técnica de ajuste descrita neste estudo e a recomendação de apenas uma equação de afilamento para árvores de Pinus taeda de diversas idades pode facilitar o trabalho de inventário, modelagem e planejamento da produção florestal de empresas do setor.

\section{CONCLUSÕES}

O polinômio do quinto grau, com os coeficientes $b_{1} \mathrm{e}$ $b_{5}$ ajustados como função linear da idade, apresenta o melhor desempenho estatístico, realismo biológico e conformidade com o conhecimento teórico sobre a forma dos troncos das árvores de Pinus taeda.

A técnica de ajuste do polinômio do quinto grau, com os coeficientes $b_{1}$ e $b_{5}$ ajustados como função linear da idade, permite que apenas uma equação de afilamento produza diversos perfis do tronco de árvores de Pinus taeda, conforme a idade. Dessa forma, apenas uma equação de afilamento pode estimar diâmetros ao longo do tronco de árvores de diferentes formas, com precisão e exatidão.

\section{AGRADECIMENTOS}

Os autores gostariam de agradecer à Universidade do Oeste de Santa Catarina (UNOESC) Campus de Xanxerê, ao Programa de Bolsas Universitárias de Santa Catarina (UNIEDU) e a empresa Juliana Florestal Ltda., do grupo Frameport.

\section{REFERÊNCIAS}

ALVARES, C.A. et al. Köppen's climate classification map for Brazil. Meteorologische Zeitschrift, v.22, n.6, p.711-728, 2013.

AHRENS, S.; HOLBERT, D. Uma função para forma de tronco e volume de Pinus taeda L. Boletim de Pesquisa Florestal, n.3, p.37$68,1981$.

COSTA, E.A. et al. Função de afilamento e sortimentos de madeira para Araucaria angustifolia. Ciência Florestal, v.26, n.2, p.523- 


\section{3, 2016.}

DAVID, H.C. et al. Critérios de estratificação para o ajuste de funções de afilamento em fustes de pinus. Pesquisa Florestal Brasileira, v.34, n.79, p.197-206, 2014.

EISFELD, R.L. et al. Modelo de Hradetzky aplicado à estimativa do volume total para Araucaria angustifolia (Bert.) 0 . Ktze. Ambiência, v.4, n.1, p.51-66, 2008.

FAVALESSA, C.M.C. et al.Equações de sortimentos para Tectona grandis na região Centro-Sul de Mato Grosso. Pesquisa Florestal Brasileira, v.32, n.72, p.389-399, 2012a.

FAVALESSA, C.M.C. et al. Funções de afilamento não segmentadas e segmentadas para Tectona grandis na região Centro-Sul matogrossense. Pesquisa Florestal Brasileira, v.32, n.72, p.373$387,2012 \mathrm{~b}$.

FIGUEIREDO, E.O. et al. Seleção de modelos polinomiais para representar o perfil e volume do fuste de Tectona grandis L.f. Acta Amazonica, v.36, n.4, p.465-482, 2006.

FIGUEIREDO FILHO, A. et al. Dinâmica do afilamento do tronco e da produção de madeira em plantios de Araucaria angustifolia. Cerne, v.20, n.4, p.595-603, 2014.

FIGUEIREDO FILHO, A. et al. Efeito da idade no afilamento e sortimento em povoamentos de Araucaria angustifolia. Floresta e Ambiente, v.22, n.1, p.50-59, 2015.

HUBER, P.J. Robust estimation of a location parameter. The Annals of Mathematical Statistics, v.35, n.1, p.73-101, 1964.

$\mathrm{HUSCH}$, B. et al. Forest mensuration. 4.ed. Hoboken: John Wiley \& Sons, 2003.

IBGE - INSTITUTO BRASILEIRO DE GEOGRAFIA E ESTATÍSTICA. Manuais técnicos em geociências: Manual Técnico de Pedologia n.4. 2ed. Rio de Janeiro: IBGE, 2007.

KOHLER, S.V. et al. Evolution of tree stem taper in Pinus taeda stands. Ciência Rural, v.46, n.7, p.1185-1191, 2016.

KOHLER, S.V. et al. Modelos de Afilamento para Pinus taeda por classe de idade. Floresta e Ambiente, v.20, n.4, p.470-479, 2013.

LANSSANOVA, L.R. et al. Avaliação de funções de afilamento para estimativa de diâmetro de espécies florestais comerciais do bioma amazônico mato-grossense. Floresta, v.43, n.2, p.215-224, 2013.

MARTINS, A.P.M. et al. Efeito da idade na forma do fuste de Araucaria angustifolia na região Centro-Sul do Paraná. Pesquisa Florestal Brasileira, v.37, n.90, p.109-117, 2017.

MIGUEL, E.P. et al. Modelos polinomiais para representar o perfil e o volume do fuste de Eucalyptus urophylla na região Norte do Estado de Goiás. Floresta, v.41, n.2, p.355-368, 2011.

MYERS, R.H. Classical and modern regression with applications. Boston: Duxbury Press, 1986.

NICOLETTI, M.F. et al. Equações hipsométricas, volumétricas e funções de afilamento para Pinus spp. Revista de Ciências
Agroveterinárias, v.19, n.4, p.474-482, 2020.

PARRESOL, B.R. Modeling multiplicative error variance: an example predicting tree diameter from stump dimensions in baldcypress. Forest Science, v.39, n.4, p.670-679, 1993.

SAS. SAS OnDemand for Academics. Cary: SAS Institute Inc., 2021.

SILVA, S.A. et al. Funções de afilamento para Pinus elliottii Engelm. proveniente de condução de regeneração natural. BIOFIX Scientific Journal, v.3, n.1, p.130-136, 2018.

SOUZA, C.A.M. et al. Modelos de afilamento para o sortimento do fuste de Pinus taeda L. Ciência Rural, v.38, n.9, p.2506-2511, 2008.

SOUZA, C.A.M. et al. Eficiência de um modelo de afilamento ajustado sem e com estratificação por classe de quociente de forma para formação dos sortimentos de Pinus taeda L. Ciência Florestal, v.22, n.1, p.125-135, 2012.

STEPKA, T.F. et al. Modelos volumétricos e funções de afilamento para Pinus taeda L. na região dos Campos Gerais, Paraná, Brasil. Espacios, v.38, n.21, p.26, 2017.

TÉO, S.J. et al. Stem taper equation with extensive applicability to several age classes of Pinus taeda L. Floresta, v.48, n.4, p.471-482, 2018a.

TÉO, S.J. et al. Modelos de afilamento para Pinus elliottii em diferentes idades, na região de Caçador, SC. Floresta, v.43, n.3, p.439-452, 2013.

TÉO, S.J. et al. Modelos de afilamento e forma dos fustes de eucalipto, em diferentes qualidades de sítio. Nativa, v.6, p.831$841,2018 b$.

TÉO, S.J. Modelagem do crescimento e produção de árvore individual independente da distância, para Pinus taeda L., na região Meio Oeste do estado de Santa Catarina. 2017. 272p. (Tese de doutorado).

TERRA, D.L.C.V. et al. Identidade de modelos volumétricos e de afilamento para Corymbia citriodora. Advances in Forestry Science, v.5, n.1, p.269-274, 2018.

TONG, Q.J.; ZHANG, S.Y. Stem form variations in the natural stands of major commercial softwoods in eastern Canada. Forest Ecology and Management, v.256, n.6, p.1303-1310, 2008.

Recebido em 28-07-2021 Aceito em 31-08-2021 